\title{
飞轮扰动下大口径长焦距光学成像系统的视轴误差 的分析与试验*
}

\author{
刘瑞婧 ${ }^{1,2}$ 金 光 ${ }^{1}$ 郭金生 $^{3}$ 李叶文 ${ }^{1,2}$ 李宗轩 ${ }^{1}$ \\ (1. 中国科学院大学长春光学精密机械与物理研究所 长春 130033; \\ 2. 中国科学院大学 北京 100039 ;
}

3. 哈尔滨工业大学卫星技术研究所 哈尔滨 150001)

\begin{abstract}
摘要: 为了分析飞轮产生的微振动对高分辨率卫星成像质量的影响, 建立了某高分辨率卫星的结构动力学模型, 测量飞轮 在轨工作时产生的微振动, 并基于整星层次计算卫星在飞轮扰动下视轴误差。计算结果表明该高分辨率卫星光学成像系统 的视轴误差的关键模态为第 8 与 9 阶模态, 主镜与次镜对视轴误差贡献最大, 可以优化主镜与次镜相关结构参数提高光学 卫星的成像质量。为验证整星结构设计与微振动分析优化的准确性与合理性, 针对整星初样开展地面微振动试验, 该卫星 在轨姿控系统为偏置动量轮设计, 通过星上 $Z / Y$ 轴动量轮模拟在轨实际工作转速, 分别在卫星悬吊状态以及固支状态下测 量了卫星颤振情况。分析与试验结果表明: 整星微振动时角位移的仿真计算结果与试验结果相比, 其相对误差 $\alpha$ 为 $11.13 \%$, 绝对值为 0.009 94", 小于 $0.01 "$, 初步认为在轨遥感图像质量不会受微振动影响而退化, 并为光学遥感卫星的微振动设计 提供了参考。
\end{abstract}

关键词: 微振动; 飞轮; 遥感卫星; 结构动力学; 视轴误差

中图分类号: V19

\section{Analysis and Test of Line-of-sight Error of Large-aperture Long Focal Length Optical Imaging System under Reaction wheel Disturbance}

\author{
LIU Ruijing $^{1,2}$ JIN Guang ${ }^{1}$ GUO Jinsheng ${ }^{3}$ LI Yewen ${ }^{1,2}$ LI Zongxuan ${ }^{1}$ \\ (1. Changchun Institute of Optics, Fine Mechanics and Physics, Chinese Academy of Sciences, \\ Changchun 130033; \\ 2. University of Chinese Academy of Sciences, Beijing 100039; \\ 3. Research Center of Satellite Technology, Harbin Institute of Tech, Harbin 150001)
}

\begin{abstract}
In order to analyze the influence of the jitter on the high resolution satellites' imaging quality, which is generated by the reaction wheel, a structural dynamic model is established to simulate the jitter effect of a high-resolution satellite on orbit. In addition, the line-of-sight (LOS) error of the optical imaging system is calculated under disturbance. The calculation results show that the critical modes of the LOS error are the 8th and 9th mode, respectively. Besides, the LOS error is deteriorated by the primary and second mirror to the largest extent, whose structural parameters can be optimized to improve the imaging quality of the satellite. To verify the accuracy and rationality of the structural design of the satellite as well as the optimization process of the jittering, an on-ground jitter simulation is performed on the prototype of the satellite, whose attitude control system is designed with the offset momentum. The actual on-orbit speed is simulated by the $Z / Y$ axis reaction wheel on the satellite, and then the jitter is obtained when the prototype is under suspended and fixed boundary conditions, respectively. Finally, we can make a comparison between the calculation and simulation results of the angular displacement of the satellite's jitter, and it indicates that the relative error $\alpha$ is $11.13 \%$, and the absolute value is 0.00994 arcsecond. With a value less than 0.01 arcsecond, we can safely draw a conclusion that the imaging quality of the remote sensing camera is not degraded by jitter. This paper provides a reference for the jitter design of the optical remote sensing satellites.
\end{abstract}

Key words: jitter; reaction wheel; remote sensing satellite; structural dynamics; line-of-sight error

* 国家科技重大专项(2016YFB0501202) 和吉林省科技发展计划 (20180414066GH) 资助项目。20190709 收到初稿, 20190929 收到修改稿 


\section{0 前言}

随着航天技术的迅猛发展, 空间相机对地观测 成像时要求成像信息更加清晰直观, 这就要求空间 相机的分辨率越来越高。为了实现高空间分辨率, 空间光学相机的焦距与口径越来越大，从而导致空 间相机的重量越来越大, 但是空间光学遥感器发射 运载条件以及重量都有极为严格的要求, 所以要对 相机进行轻量化设计, 空间相机光机系统的刚度受 重量限制提升空间有限，导致空间相机在轨工作时 易受到星上转动部件产生的微振动的影响。

星上转动部件正常工作时造成的微振动的振幅 非常小, 一般不会对航天器结构产生破坏性影响。 但是, 它却可以导致平台颤振、降低其载荷光轴指 向的稳定度和指向精度。在空间微重力环境下星上 各类扰振源 (太阳翼驱动机构、姿态控制飞轮等)都 会造成光学成像系统的视轴振动 (Line-of-sight error, LOS error), 即光学像面相对成像探测器 “抖 动” ${ }^{[1]}$ 。其中姿态控制飞轮是主要的扰振源。在航 天器的飞行过程中, 飞轮内部转子会持续高速旋转 并产生频率成分复杂的扰振力与扰振力矩, 引起颤 振, 降低光学系统的在轨动态传递函数, 造成像质 退化。

飞轮颤振已经制约着航天技术的发展, 因此各 国均对飞轮微振动问题展开广泛研究。国外大多关 注于隔振平台的设计以及新型飞轮的设计, 英国南 安普顿大学的 ZHANG 提出了基于弹性支承的隔振 飞轮的设计方案 ${ }^{[2]}$, Millennium Engineering and Integration Company 的 NAGABHUSHAN 等研制出 多组轮体组合的新型智能飞轮 ${ }^{[3]}$, 国内对于微振动 的研究尚处于起步阶段 ${ }^{[4]}$, 目前关于微振动的研究 工作主要集中在理论建模方面, 部分实验研究则主 要是关于隔振装置的设计与测试, 例如, 清华大学 与北京控制研究所等单位合作提出了一种由被动隔 振杆单元与调谐质量阻尼器构成的复合隔振方法, 这种隔振平台的设计隔振效果较好, 但是会引入刚 度不确定性，并且使整星相对质量变大 ${ }^{[5]}$ 。

微振动问题已经成为降低成像质量的关键因 素, 因此亟需在整星层次上开展微振动的光机集成 研究, 本文考虑飞轮以及桁架支撑结构相互耦合对 成像的影响, 在整星层次上利用光机集成的分析方 法对高分辨率空间相机的颤振影响进行分析, 建立 了整星结构动力学模型; 对 $Z$ 轴飞轮以及 $Y$ 轴飞轮 进行扰振测试试验, 得到飞轮的扰振特性, 并且将 实际测得的飞轮振动数据与整星结构进行耦合, 仿
真计算得出在飞轮扰振下整星的视轴(LOS)误差; 为 验证整星结构设计与微振动分析优化的准确性与合 理性, 针对整星初样开展地面微振动试验, 试验测 量了在 $Z$ 向以及 $Y$ 向飞轮作用下整星颤振情况, 并 将仿真结果与试验结果进行对比分析，对于整星结 构布局以及光学系统设计具有指导性意义。

\section{1 飞轮微振动产生机理}

\section{1 微振动产生机理}

卫星在轨运行工作过程中受到星上各类扰振源 的干扰, 如飞轮、陀螺、驱动机构等。受转子动静 不平衡、驱动电机误差、轴承缺陷等因素的影响, 飞轮正常工作时会产生复杂的谐波扰动及噪声, 引 起卫星姿态抖动与结构振动, 姿控飞轮是主要的扰 振源 ${ }^{[6]}$ 。

基于角动量守恒原理, 飞轮通过调节转子转速, 与卫星本体之间进行角动量交换，实现卫星姿态控 制。作为典型的高速旋转部件, 动量轮在提供必要 的控制力矩的同时也会产生扰动, 影响成像质量 ${ }^{[7]}$ 。 动量轮的扰动产生主要来源于两种机理: (1)主动扰 动力, 由于旋转产生的, 主要包括动量轮的动不平 衡、滚动轴承和电机扰动等; (2)结构扰动力, 主动 扰动力引起的动量轮内部结构响应形成的对动量轮 外部的扰动力。两类扰动力关系如图 1 所示 ${ }^{[6]}$ 。

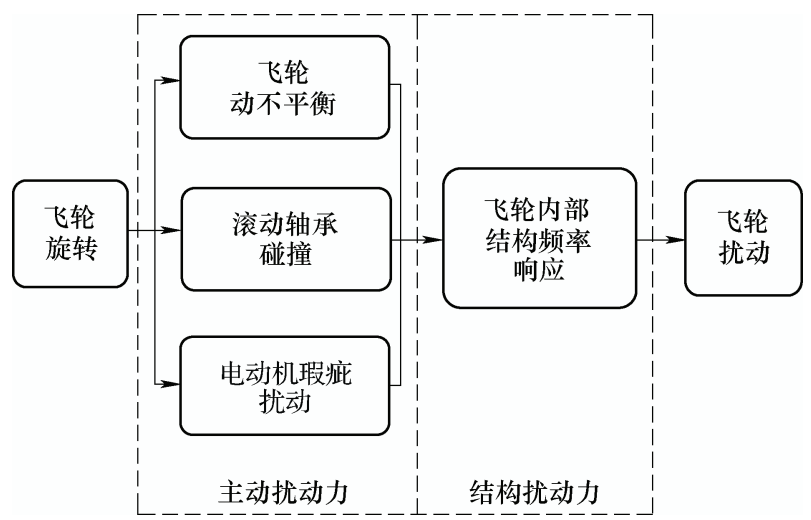

图 1 动量轮扰动机理

\section{2 飞轮扰振模型}

根据飞轮的扰振特性可建立飞轮的扰振模型, 如公式(1)所示

$$
m(t)=C \Omega^{2} \sin (2 \pi h \Omega t+\alpha)
$$

式中 $m(t)$ 一一扰动力或者扰动力矩;

$$
\begin{aligned}
& C \text { 一扰动幅值; } \\
& \Omega \text { —飞轮转速; } \\
& h \text { —谐波数; } \\
& \alpha \text { 一随机相位 }(0,2 \pi) \text { 。 }
\end{aligned}
$$


动量轮结构示意图如图 2 所示, 以动量轮的 几何中心为坐标系原点, 三轴指向相对于动量轮 初始位置保持不变。用动量轮质心偏离其几何中 心的位置和距离表示静态不平衡 $U_{s}=m_{s} r_{s}$ 和动 态不平衡 $U_{d}=2 m_{d} r_{d} h$ 的影响, 轴承的柔性和阻 尼的影响用一个线形弹簧和阻尼代表。其中各参 数含义如图 2 所示, 距离转轴为 $r_{d}$ 的两个点质量 $m_{d}$, 沿旋转轴方向距离为 $h$, 点质量 $m_{s}$ 与动量 轮转轴处距离为 $r_{s}$ 。 $k$ 和 $c$ 分别为轴承支持刚度 和阻尼。

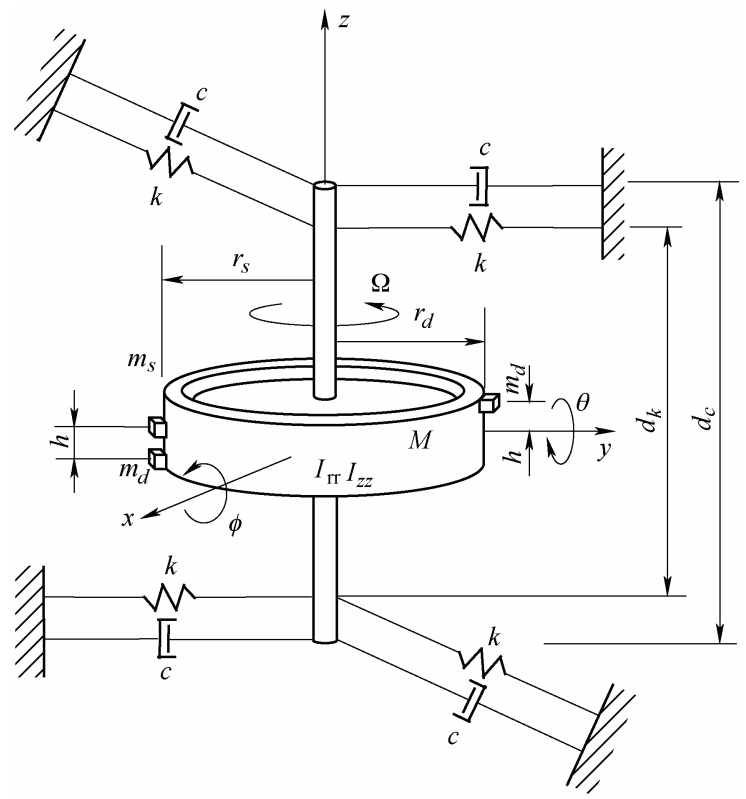

图 2 动量轮弹性振动弹簧一阻尼器模型

对于动量轮轴承的转动支撑, 其刚度和阻尼为

$$
k_{\theta}=k d_{k}^{2}, \quad c_{\theta}=c d_{c}^{2}
$$

其中 $d_{k}$ 与 $d_{c}$ 几何意义如图 2 所示。

针对飞轮的扰振模型要有如下假设, 不平衡质量 与总质量相比是可以忽略的, 即 $M_{t} \gg m_{s}, m_{d}, I_{r r}$, $I_{z z} \gg \tilde{I}$, 其中 $M_{t}=M+m_{s}+2 m_{d}, \tilde{I}=2 m_{d}^{2} r_{d}^{2}+m_{s}^{2} r_{s}^{2}$

$$
\begin{gathered}
\left(\begin{array}{cc}
M_{t} & 0 \\
0 & M_{t}
\end{array}\right)\left(\begin{array}{l}
\ddot{x} \\
\ddot{y}
\end{array}\right)+\left(\begin{array}{cc}
c & 0 \\
0 & c
\end{array}\right)\left(\begin{array}{c}
\dot{x} \\
\dot{y}
\end{array}\right)+\left(\begin{array}{ll}
k & 0 \\
0 & k
\end{array}\right)\left(\begin{array}{l}
x \\
y
\end{array}\right)= \\
\sum_{i=1}^{n} C_{r a d i} \Omega^{2}\left(\begin{array}{c}
-\sin \left(h_{\text {radi }} \Omega t\right) \\
\cos \left(h_{\text {radi }} \Omega t\right)
\end{array}\right) \\
\left(\begin{array}{cc}
I_{r r} & \frac{1}{2} \tilde{I} \sin (2 \Omega t) \\
\frac{1}{2} \tilde{I} \sin (2 \Omega t) & I_{r r}
\end{array}\right)\left(\begin{array}{c}
\ddot{\theta} \\
\ddot{\Phi}
\end{array}\right)+\Omega \\
\left(\begin{array}{cc}
\frac{C_{\theta}}{\Omega}-\tilde{I} \sin (2 \Omega t) & I_{z z} \\
-I_{z z} & \frac{C_{\theta}}{\Omega}-\tilde{I} \sin (2 \Omega t)
\end{array}\right)\left(\begin{array}{c}
\dot{\theta} \\
\dot{\Phi}
\end{array}\right)+
\end{gathered}
$$

$$
\left(\begin{array}{cc}
k_{\theta} & 0 \\
0 & k_{\theta}
\end{array}\right)\left(\begin{array}{c}
\theta \\
\Phi
\end{array}\right)=\sum_{i=1}^{n} C_{t o r i} \Omega^{2}\left(\begin{array}{c}
\cos \left(h_{\text {tori }} \Omega t\right) \\
\sin \left(h_{\text {tori }} \Omega t\right)
\end{array}\right)
$$

公式(1)计算的扰动力与扰动力矩代入式(3)、(4) 中, 飞轮产生的扰动可通过式(5)近似的转换为传递 扰动 ${ }^{[8]}$

$$
\left(\begin{array}{l}
F_{x} \\
F_{y} \\
C_{x} \\
C_{y}
\end{array}\right)=\left(\begin{array}{c}
k x \\
k y \\
k_{\theta} \theta \\
k_{\Phi} \Phi
\end{array}\right)
$$

\section{2 整星微振动结构动力学分析}

光学遥感卫星在轨工作期间易受到微振动的影 响, 这种微振动能量小, 频带宽, 会引起相机结构 和光学系统各个零件内部的微变形以及微位移。为 进行整星微振动分析, 建立整星有限元模型, 并将 实际测得的飞轮扰动数据作为输入, 仿真计算卫星 在轨工作时整星角位移, 并根据整星在轨实际工作 情况进行地面整星微振动试验, 并将仿真结果与试 验结果对比, 保证分析以及试验的准确性。

\section{1 整星微振动力学模型}

卫星的运载、发射以及工作环境较为恶劣, 并 且通常情况下, 光学遥感卫星结构复杂, 其相机内 部支撑结构与反射镜难以用简单的板壳单元等效, 为预先确切计算光学卫星在轨工作状况, 采用有限 元的方法进行分析计算, 以提高计算效率以及准确 性, 整星的动力学模型可以由式(6)来描述

$$
\boldsymbol{M} \ddot{\boldsymbol{u}}+\boldsymbol{C} \dot{\boldsymbol{u}}+\boldsymbol{K} \boldsymbol{u}=\boldsymbol{F}
$$

式中, $M$ 为 $R_{n \times n}$ 维质量矩阵; $\boldsymbol{C}$ 为 $R_{n \times n}$ 维阻尼矩阵; $\boldsymbol{K}$ 为 $R_{n \times n}$ 维刚度矩阵; 其中 $n$ 为自由度, $R$ 为实数集; $\ddot{\boldsymbol{u}} 、 \dot{\boldsymbol{u}} 、 \boldsymbol{u}$ 为结构响应的 $n \times 1$ 维列矢量, 分别表示加速 度、速度、位移; $\boldsymbol{F}$ 为 $n \times 1$ 维列矢量, 表示输入载荷。

由于飞轮产生的微振动量级很小, 所以近似认 为光学元件的振动与视轴抖动之间的关系是线性 的, 根据模态分析理论引入模态变换 $\boldsymbol{u}=\boldsymbol{\Phi} \boldsymbol{x}(\boldsymbol{\Phi}$ 为 $R_{n \times n}$ 的模态矩阵, $x$ 为模态坐标)将物理坐标转换成 模态坐标, 对式(6)解耦, 通过线性变换得到物理坐 标响应, 即每个振型的响应

$\left(\begin{array}{lll}\ddots & & 0 \\ & m_{i} & \\ 0 & & \ddots\end{array}\right) \ddot{u}+\left(\begin{array}{lll}\ddots & & 0 \\ & c_{i} & \\ 0 & & \ddots\end{array}\right) \dot{u}+\left(\begin{array}{lll}\ddots & & 0 \\ & k_{i} & \\ 0 & & \ddots\end{array}\right)=\left(\begin{array}{c}\vdots \\ f_{i} \\ \vdots\end{array}\right)$

式中, $m_{i}, c_{i}, k_{i}(i=1, \cdots, n)$ 分别称为系统的模态质 量、模态阻尼、模态刚度 ${ }^{[9]}$ 。

\section{2 整星微振动有限元模型}

为了进行以飞轮微振动试验为基础的整星结构 
动力学分析, 需建立整星的有限元模型。整星模型 坐标系的 $X$ 轴为飞行方向, $Z$ 轴为光轴方向, $Y$ 轴 方向遵循右手定则 ${ }^{[6]}$ 。

以某大口径长焦距空间相机为例, 如图 3 所示, 首先通过 UG 对整星进行三维建模, 利用 Hypermesh 的几何处理和网格划分功能对某卫星的整星结构建 立有限元模型, 并进行材料和单元属性的定义, 通 过 MSC.Patran/Nastran 对模型施加载荷以及建立边 界条件(计算自由模态), 并对该卫星有限元模型进 行计算分析。该空间相机有限元模型如图 4 所示, 共计 135186 个单元，192 191 个节点。

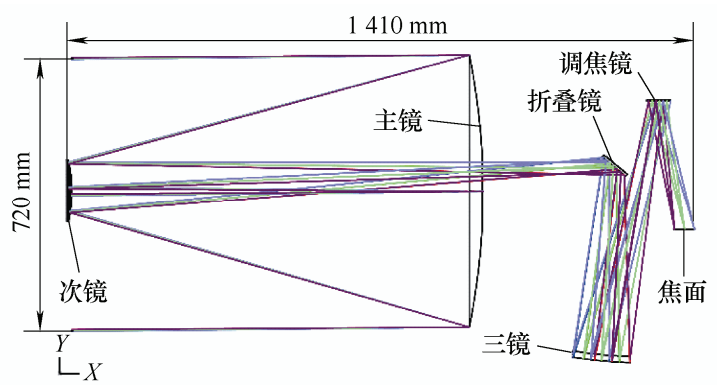

图 3 某空间相机光学系统示意图

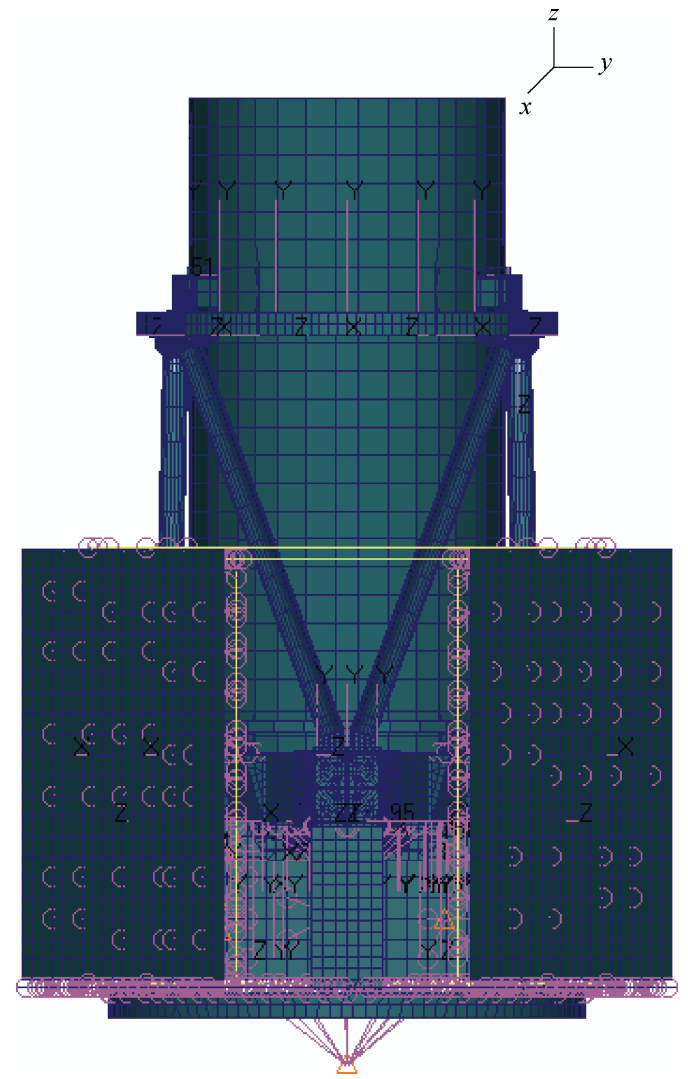

图 4 整星有限元模型

3 飞轮振动下整星的视轴误差

\section{1 视轴误差}

飞轮工作过程中产生的微振动为宽频随机振动,
对于随机振动的有限元分析首先计算模型固有频率 与振型, 模态分析可得到频率响应函数(Frequency response function,FRF), 通过 PSD 输入与 FRF 即可计 算得出光学系统的 LOS 的 PSD 响应函数 $\left(P S D_{\text {resp }}\right)$, 其包围面积的平方根是随机响应的均方根值 $R M S_{\text {resp }}$, 如图 5 所示, 表达式如式(8)、(9)所示 ${ }^{[10]}$

$$
\begin{aligned}
& P S D_{\text {resp }}=F R F_{\text {resp }}^{2} P S D_{\text {input }} \\
& 1 \sigma_{\text {resp }}=R M S_{\text {resp }}=\sqrt{\text { Area }}
\end{aligned}
$$

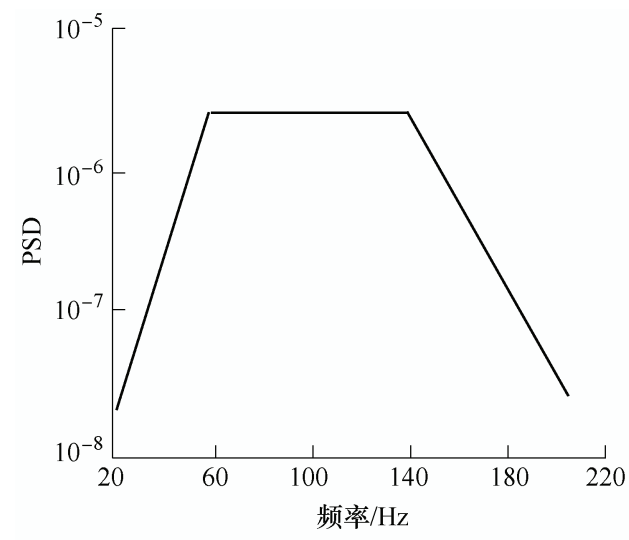

(a) PSD输入

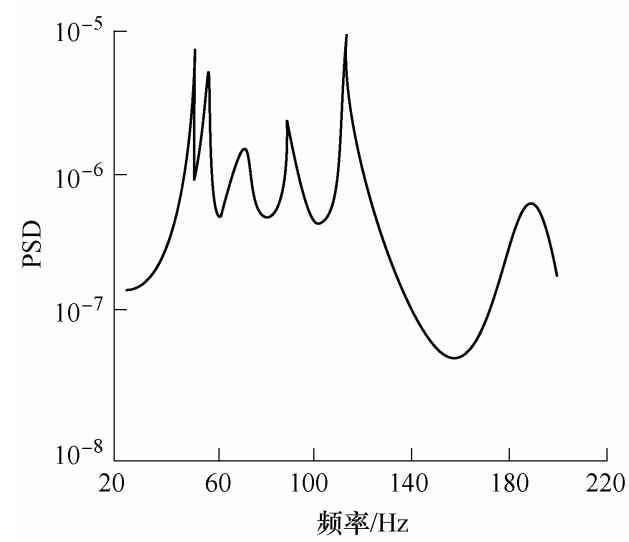

(b) FRF响应

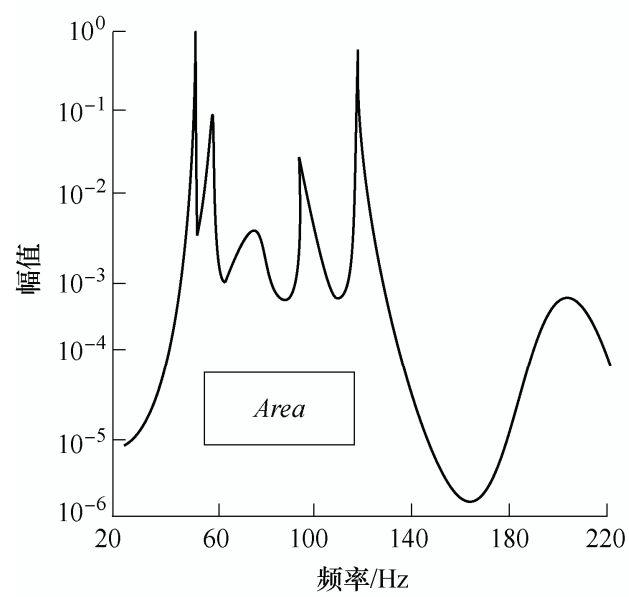

(c) PSD响应

图 5 随机振动响应分析

在星上飞轮微振动的作用下空间相机的结构产 
生的微振动, 不仅会造成空间相机光学元件的刚体 位移以及弹性变形, 导致光学成像系统的传递函数 退化, 还会造成视轴振动, 即光学像点在像面上的 横向移动, 如图 6 所示, 所以视轴误差成为评价光 学成像系统成像质量的有力指标。

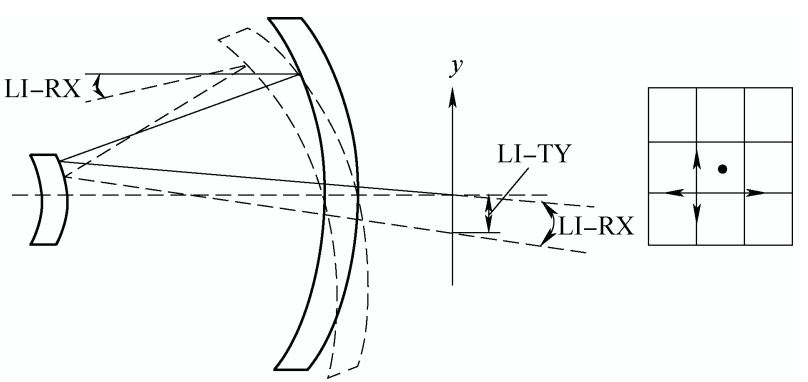

图 6 视轴误差示意图

由于结构阻尼的作用, LOS 的运动在每个频率 步长具有不同相位 $\left(\Phi_{x}, \Phi_{y}\right)$ 的 $X$ 分量与 $Y$ 分量 $\left(T_{x}\right.$, $\left.T_{y}\right)$ 。任意给定频率下, LOS 的幅值 $\left(\Delta=T_{\mathrm{v}}\right)$ 可以由 式(10) (14)计算得出, 光学成像系统在微振动的影 响下角位移 $(2 \Theta)$ 由式(11) 可得 ${ }^{[10]}$

$$
\begin{gathered}
\bar{T}_{x}=T_{x} \cos \left(\Theta+\Phi_{x}\right) \\
\bar{T}_{y}=T_{y} \cos \left(\Theta+\Phi_{y}\right) \\
\Delta^{2}=T_{v}^{2}=T_{x}^{2} \cos ^{2}\left(\Theta+\Phi_{x}\right)+T_{y}^{2} \cos ^{2}\left(\Theta+\Phi_{y}\right) \\
\frac{\mathrm{d} T_{v}}{\mathrm{~d} \Theta}=0 \\
-\tan (2 \Theta)=\frac{T_{x}^{2} \sin ^{2}\left(2 \Phi_{x}\right)+T_{y} \sin ^{2}\left(2 \Phi_{y}\right)}{T_{x}^{2} \cos ^{2}\left(2 \Phi_{x}\right)+T_{y}^{2} \cos ^{2}\left(2 \Phi_{y}\right)}
\end{gathered}
$$

\section{$3.2 飞$ 轮扰动测试过程}

飞轮扰动试验在超净环境实验室中进行, 测试 过程中将飞轮以及其支撑结构安装于气浮台的测力 平台上, 飞轮支架结构通过螺钉与飞轮固连, 测试 现场如图 7 所示, 通过飞轮振动试验测得飞轮在正 常工作时的 $X / Y / Z$ 方向的扰动力与扰动力矩, 根据 计算得出的扰动力/力矩建立飞轮的仿真模型, 与整 星的仿真模型集成建模。

\section{3 整星微振动视轴误差仿真分析计算}

整星的视轴误差通过光机集成软件 Sigfit 进行 分析计算。Sigfit 中嵌入了光线跟踪算法, 并且可以 读取 Nastran/Patran 输出的.bdf/.pch 文件, 采用基于 矢高的坐标系变换, 进行 Zernike 拟合, 可直接输 出系统在微振动作用下产生的视轴误差。Sigfit 可以 计算得出在飞轮实际扰动下视轴漂移引起的 LOS 误差以及视轴颤振引起的 LOS 误差, 测得飞轮在正常 工作过程中的扰动数据, 经过 Matlab 处理后作为 PSD 输入, 如图 8 所示, 与整星有限元模型集成分析,

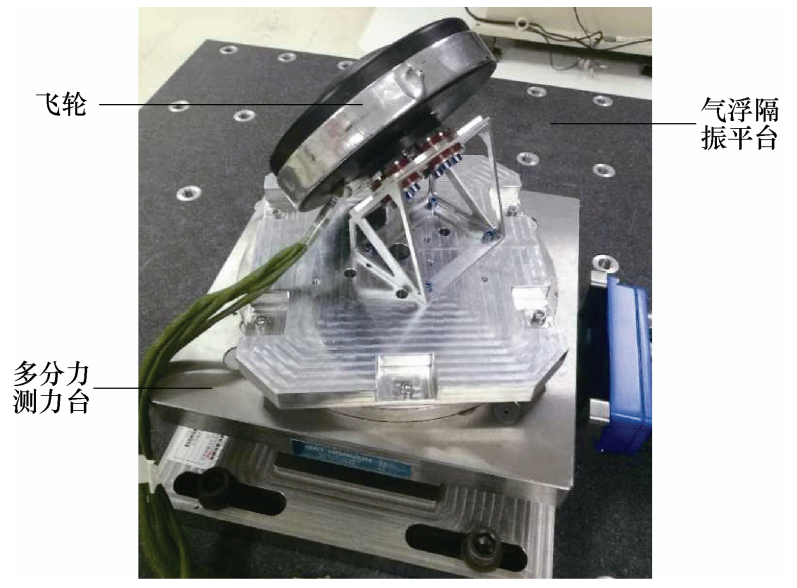

图 7 飞轮微振动测试

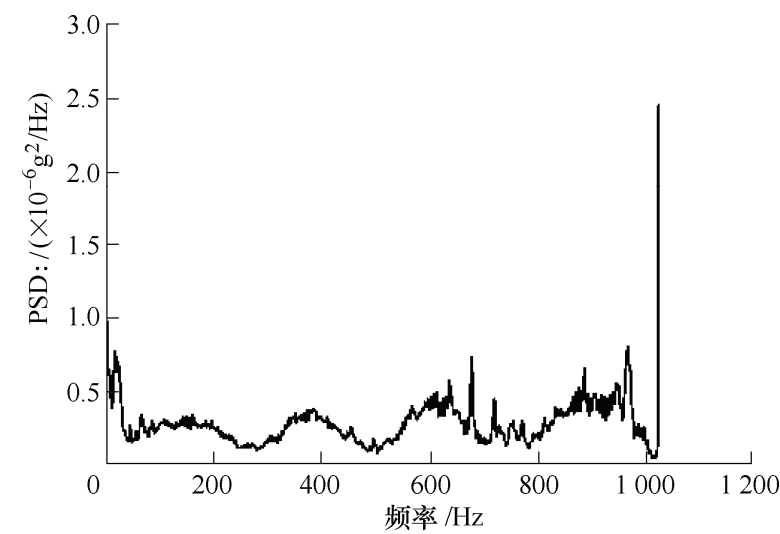

图 8 飞轮微振动 PSD 输入

由于抖动项比漂移项更重要, 这里我们只关注抖动 引起的视轴误差, 分析流程如图 9 所示。

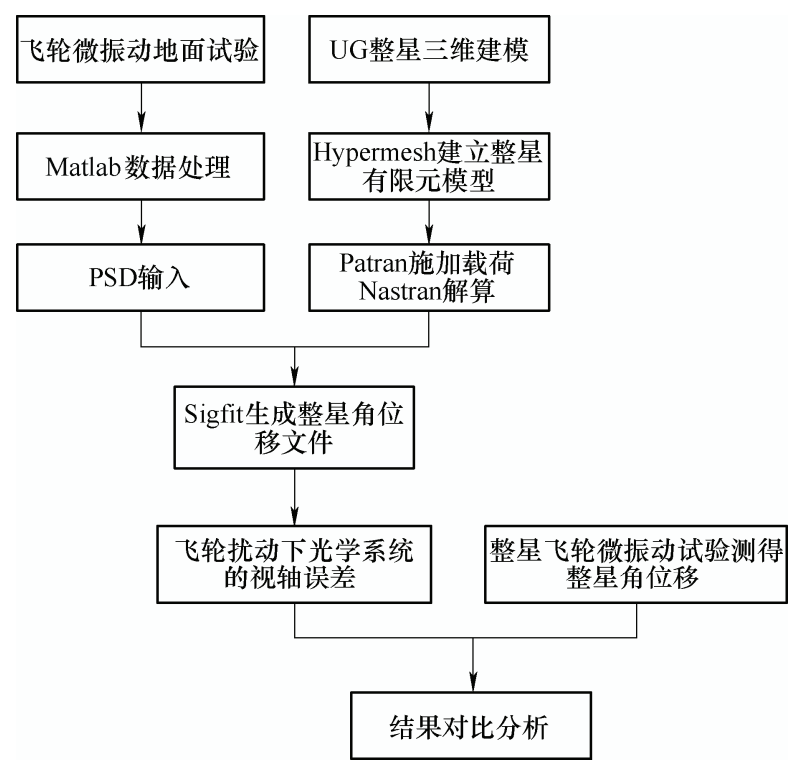

图 9 飞轮微振动下光学系统 LOS 分析流程图

光学卫星在轨工作过程中, $Z$ 向飞轮在大多数 时间里以 $1200 \mathrm{r} / \mathrm{min}$ 的转速工作, 为了分析计算整 星结构设计的合理性以及准确性, 以 $Y$ 向飞轮与 $Z$ 向飞轮同时工作的情况为例, 也就是分析振动最为 剧烈的状态下整星的视轴误差, 计算出颤振的随机 
响应的抖动分量, 如表 1 所示, 其中 LI 与 LO 分别 表示像空间与物空间, $\mathrm{TX}$ 与 $\mathrm{TY}$ 分别表示延 $X$ 轴 与 $Y$ 轴的线性位移量, 其中 LI-TV/LO-TV 分别表示 像空间在 $X Y$ 平面上线性位移矢量和 $(\mathrm{mm}), \mathrm{RX}$ 与 $\mathrm{RY}$ 则分别表示绕 $X / Y$ 轴的转动, LI-RV/LO-RV 分 别为像空间与物空间转动量矢量和 $(\mathrm{rad})$ 。

Sigfit 计算出每阶自由模态对总的 LOS 误差贡 献的百分数, 如表 2 所示, 该高分辨率光学卫星光 学系统的 LOS 关键模态为第 8 和 9 阶模态, 即对 LOS 误差贡献最大, 也就是说, 第 8 阶与第 9 阶模态是 产生视轴误差的主要原因。由于其他阶次模态贡献
较小, 这里不一一列出, 表 2 只给出了 4-10 阶模态, 前后对比观察趋势。

表 1 颤振随机响应的分析

\begin{tabular}{ccc}
\hline & 响应均方值 & 过零时间 \\
\hline 像空间 $X$ 方向平动 LI-TX/mm & $1.0190 \times 10^{-3}$ & $3.5790 \times 10^{1}$ \\
像空间 $Y$ 方向平动 LI-TY/mm & $7.4772 \times 10^{-4}$ & $3.4328 \times 10^{1}$ \\
像空间绕 $X$ 轴转动 $\mathrm{LI}-\mathrm{RX} / \mathrm{rad}$ & $7.8987 \times 10^{-7}$ & $3.4322 \times 10^{1}$ \\
像空间绕 $Y$ 轴转动 LI-RY/rad & $2.1717 \times 10^{-6}$ & $3.5508 \times 10^{1}$ \\
物空间绕 $X$ 轴转动 LO-RX/rad & $2.8700 \times 10^{-7}$ & $3.4328 \times 10^{1}$ \\
物空间绕 $Y$ 轴转动 LO-RY/rad & $3.9112 \times 10^{-7}$ & $3.5790 \times 10^{1}$ \\
\hline
\end{tabular}

表 2 各阶模态对 LOS 贡献百分比

\begin{tabular}{|c|c|c|c|c|c|c|c|c|c|c|}
\hline \multirow{3}{*}{ 模态 } & \multirow{3}{*}{ 频率/Hz } & \multicolumn{3}{|c|}{ 像方平动 } & \multicolumn{3}{|c|}{ 像方转动 } & \multicolumn{3}{|c|}{ 物方转动 } \\
\hline & & $X$ 向 & $Y$ 向 & 矢量和 & $X$ 向 & $Y$ 向 & 矢量和 & $X$ 向 & $Y$ 向 & 矢量和 \\
\hline & & LI-TX & LI-TY & LI-TV & LI-RX & LI-RY & LI-RV & LO-RX & LO-RY & LO-RV \\
\hline 4 & 0 & 0.014 & 0 & 0.008 & 0 & 0.015 & 0.013 & 0 & 0.014 & 0.008 \\
\hline 5 & 0 & 0.004 & 0.097 & 0.042 & 0.103 & 0.004 & 0.019 & 0.097 & 0.004 & 0.042 \\
\hline 6 & 0 & 0.204 & 0.027 & 0.132 & 0.028 & 0.224 & 0.195 & 0.027 & 0.204 & 0.132 \\
\hline 7 & 29.62 & 6.124 & 7.900 & 6.851 & 7.938 & 7.226 & 7.331 & 7.900 & 6.124 & 6.851 \\
\hline 8 & 30.98 & 9.414 & 61.498 & 30.728 & 61.507 & 12.679 & 19.870 & 61.498 & 9.414 & 30.728 \\
\hline 9 & 38.42 & 84.240 & 30.381 & 62.199 & 30.310 & 79.852 & 72.555 & 30.381 & 84.240 & 62.200 \\
\hline 10 & 41.18 & 0 & 0.097 & 0.040 & 0.114 & 0.001 & 0.018 & 0.097 & 0 & 0.040 \\
\hline
\end{tabular}

识别出关键模态的同时, Sigfit 同时可以得到各 光学元件对 $\operatorname{LOS}$ 误差的贡献值, 其计算结果如表 3 所示, 其中 SID(Surface ID) $1 / 2 / 3$ 分别为主镜、次镜
与三镜, DOF 表示 6 个自由度, 可以看出主镜与次 镜对 LOS 的影响最大, 可优化主镜与次镜相关结构 以及光学参数提高光学系统成像质量。

表 3 各光学镜面对 LOS 的贡献

\begin{tabular}{|c|c|c|c|c|c|c|c|}
\hline \multirow{3}{*}{ SID } & \multirow{3}{*}{$\begin{array}{l}\text { 自由度 } \\
\text { (DOF) }\end{array}$} & \multicolumn{2}{|c|}{ 像方平动 } & \multicolumn{2}{|c|}{ 像方转动 } & \multicolumn{2}{|c|}{ 物方转动 } \\
\hline & & $X$ 向 & $Y$ 向 & $X$ 向 & $Y$ 向 & $X$ 向 & $Y$ 向 \\
\hline & & LI-TX/mm & LI-TY/mm & LI-RX/rad & LI-RY/rad & LO-RX/rad & $\mathrm{LO}-\mathrm{RY} / \mathrm{rad}$ \\
\hline 1 & 1 & 2.3868 & 0 & 0 & -0.0047 & 0 & 0.0009 \\
\hline 1 & 2 & 0 & 2.3868 & 0.0021 & 0 & -0.0009 & 0 \\
\hline 1 & 3 & 0 & 0 & 0 & 0 & 0 & 0 \\
\hline 1 & 4 & 0 & 5210.7164 & 5.7557 & 0 & -2.0000 & 0.0000 \\
\hline 1 & 5 & -5210.5199 & 0 & 0 & 10.8034 & 0 & -2.0000 \\
\hline 1 & 6 & 0 & 0 & 0 & 0 & 0 & 0 \\
\hline 2 & 1 & 5.1717 & 0 & 0 & -0.0143 & 0 & 0.0020 \\
\hline 2 & 2 & 0.0000 & 5.1717 & 0.0071 & 0 & -0.0020 & 0 \\
\hline 2 & 3 & 0.0000 & 0 & 0 & 0 & 0 & 0 \\
\hline 2 & 4 & 0 & 2320.8933 & 3.2565 & 0 & -0.8908 & 0 \\
\hline 2 & 5 & -2320.8643 & 0 & 0 & 6.3886 & 0 & -0.8908 \\
\hline 2 & 6 & 0 & 0 & 0 & 0 & 0 & 0 \\
\hline 3 & 1 & -1.0000 & 0 & 0 & 0.0047 & 0 & -0.0004 \\
\hline 3 & 2 & 0 & -1.0000 & -0.0021 & 0 & 0.0004 & 0 \\
\hline 3 & 3 & 0 & 0 & 0 & 0 & 0 & 0 \\
\hline 3 & 4 & 0 & 132.2479 & 1.2995 & 0 & -0.0508 & 0 \\
\hline 3 & 5 & -132.2509 & 0 & 0 & 2.8984 & 0 & -0.0508 \\
\hline 3 & 6 & -433.5700 & 0.0022 & 0 & 2.0435 & 0 & -0.1664 \\
\hline
\end{tabular}

\section{4 飞轮微振动测试试验}

卫星在轨工作过程中, 通过与姿控飞轮进行角
动量交换实现在轨姿态控制, 飞轮在工作过程中内 部转子高速运转并产生扰动力与扰动力矩, 使卫星 产生结构振动以及姿态抖动, 产生视轴误差, 降低 成像质量。根据仿真分析结果对整星结构进行优化 
后, 在整星层次上进行飞轮微振动测试试验并进行 频率响应分析。

\section{1 整星飞轮微振动测试试验}

整星微振动测试试验在微振动专用测试实验室 中进行, 卫星垂直悬吊, 模仿整星在轨状态, 光纤 陀螺安装于整星的空间相机上, 测量飞轮正常工作 状态下的颤振情况, 采样频率为 $1000 \mathrm{~Hz}$, 整星微 振动测试如图 10 所示。该微振动试验首先测量背景 噪声, 根据飞轮实际在轨控制设计策略, 分别测量 了 $Z$ 轴飞轮转速为 $1200 \mathrm{r} / \mathrm{min} 、 1550 \mathrm{r} / \mathrm{min} 、 2078$ $\mathrm{r} / \mathrm{min}$ 时整星状态, 并记录光纤陀螺相应数据, 将 $Y$ 轴飞轮停止后, $Y$ 轴飞轮单独加速至 $600 \mathrm{r} / \mathrm{min}$, 记 录数据后 $Y$ 轴飞轮停止工作。最后测量 $Z$ 轴飞轮加 速至 $1200 \mathrm{r} / \mathrm{min} 、 Y$ 轴飞轮加速至 $600 \mathrm{r} / \mathrm{min}$, 微振 动测试试验测试过程如图 11 所示。

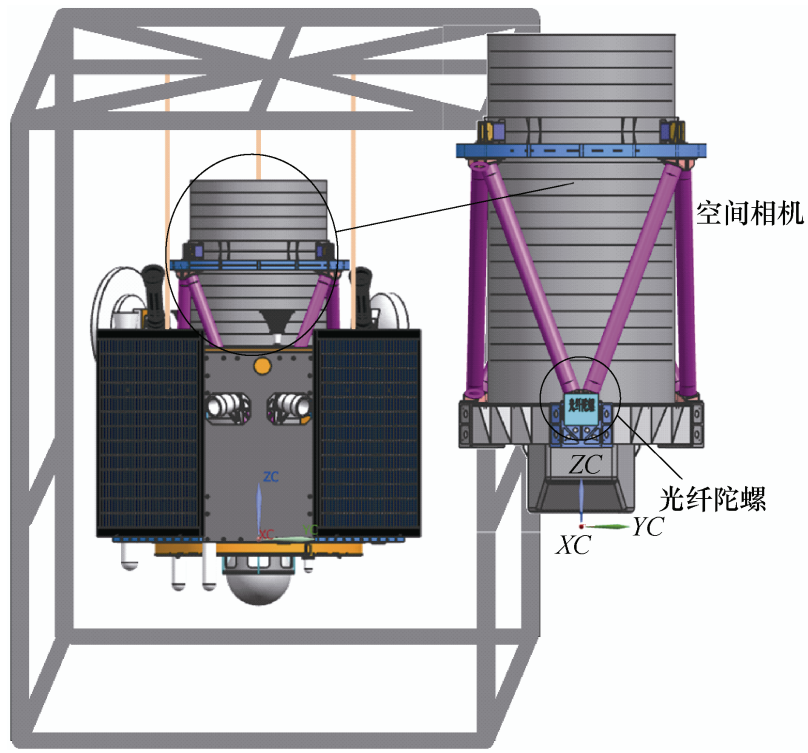

图 10 整星微振动测试示意图

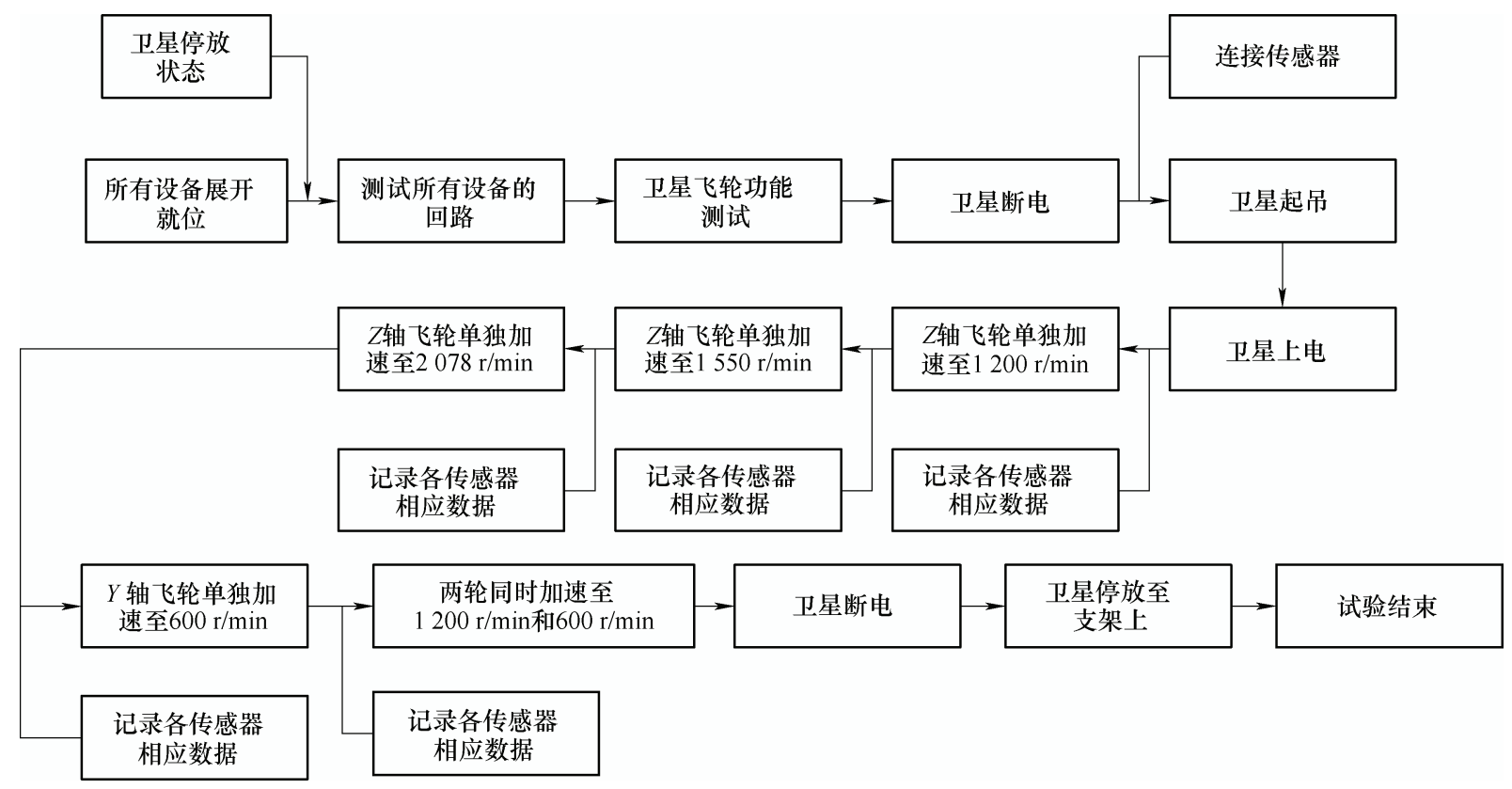

图 11 微振动测试过程

4.2 整星飞轮微振动测试结果分析

根据微振动试验测试流程完成试验, 并记录各

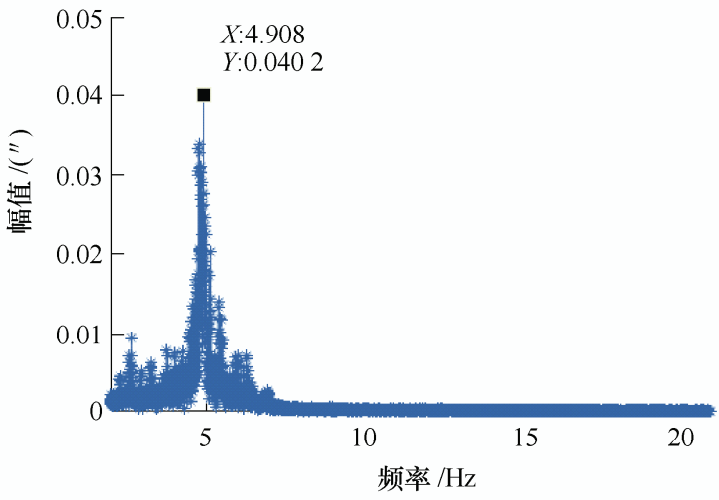

(a) 背景噪声下整星角位移
工况试验结果并对其进行分析。分析结果如图 12 所示。

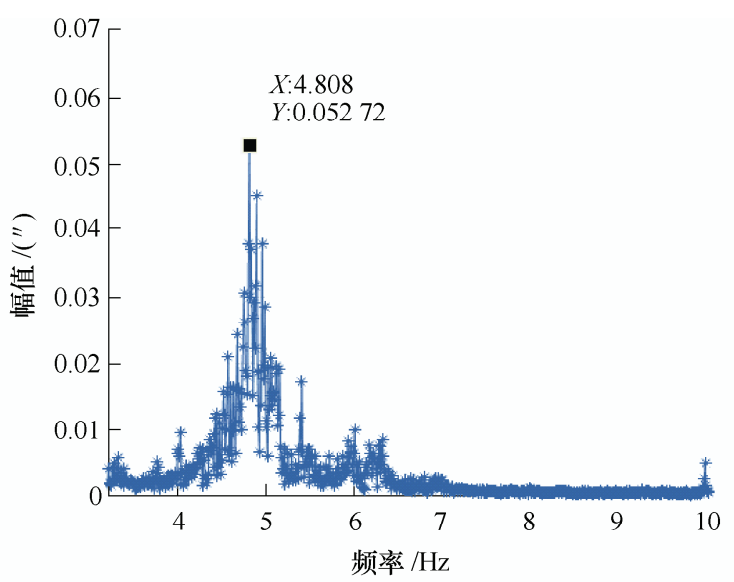

(b) $Y$ 向飞轮转速为 $600 \mathrm{r} / \mathrm{min}$ 时整星角位移 


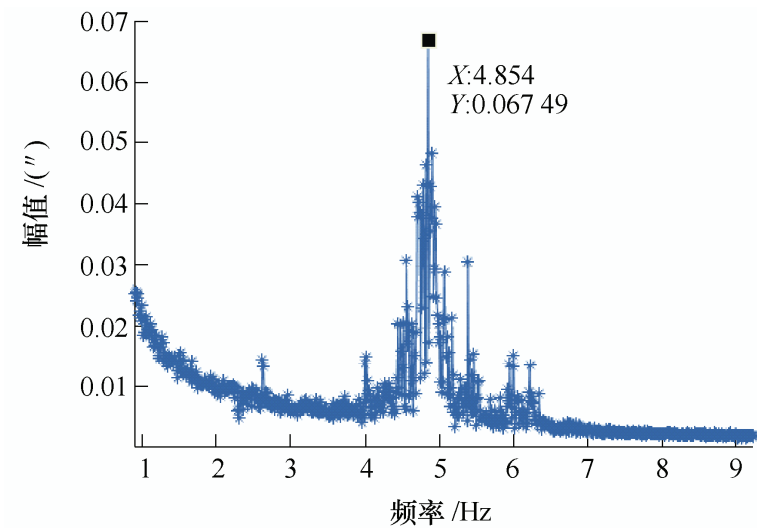

(c) $Z$ 向飞轮转速为 $1200 \mathrm{r} / \mathrm{min}$ 时整星角位移

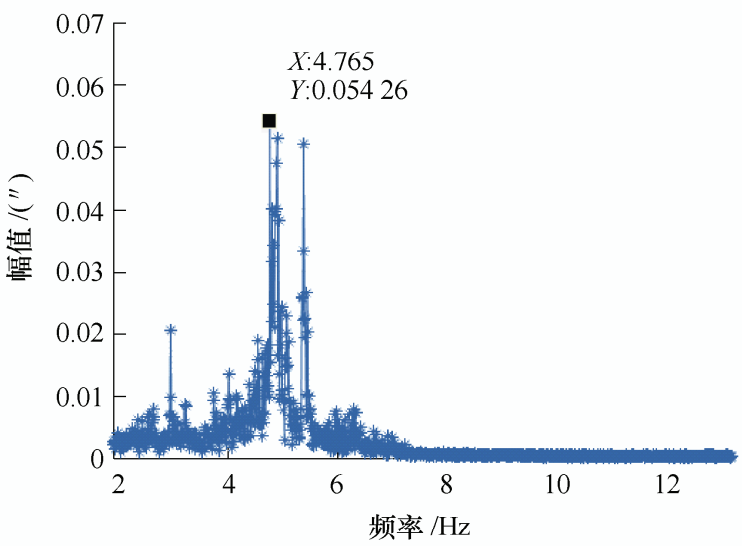

(e) $Z$ 向飞轮转速为 $2078 \mathrm{r} / \mathrm{min}$ 时整星角位移

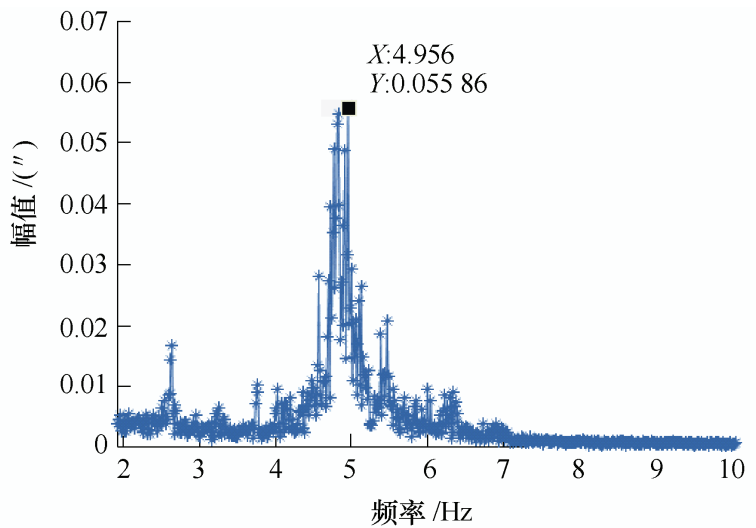

(d) $Z$ 向飞轮转速为 $1550 \mathrm{r} / \mathrm{min}$ 时整星角位移

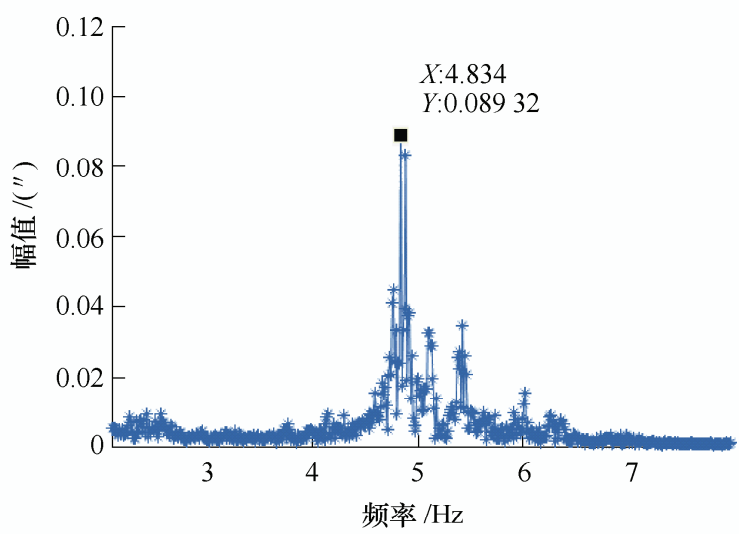

(f) $Y$ 向飞轮转速为 $600 \mathrm{r} / \mathrm{min} Z$ 轴飞轮转速为 $1200 \mathrm{r} / \mathrm{min}$ 时整星角位移

图 12 卫星悬吊状态下整星角位移

各种工况频率分析计算结果如图 12 所示, 其横 坐标为频率 $(\mathrm{Hz})$, 纵坐标为角位移 (")。由结果图分析 可知, (1) 在各种背景噪声的影响下整星的角位移为 0.040 2"(图 12a); (2) $Y$ 向飞轮以 $600 \mathrm{r} / \mathrm{min}$ 单独工作 时,整星角位移为 0.06749 "(图 12b); (3) $Z$ 向飞轮以 $1200 \mathrm{r} / \mathrm{min}$ 的转速单独工作时, 整星角位移为 $0.06749^{\prime \prime}$ (图 12c); (4) $Z$ 向飞轮以 $1550 \mathrm{r} / \mathrm{min}$ 的转速
单独工作时, 整星角位移为 $0.05586^{\prime \prime}$; (5) $Z$ 向飞轮 以 $2078 \mathrm{r} / \mathrm{min}$ 的转速单独工作时, 整星角位移为 0.05426 "(图 12e); (6) $Y$ 向与 $Z$ 向飞轮共同工作时,整 星角位为 0.08932 , 此时整星颤振最为剧烈(图 12f)。 由以上频谱分析可知: 整星悬吊状态下，在 $Z / Y$ 向飞 轮工作过程中存在 $4 \sim 5 \mathrm{~Hz}$ 的低频信号。接下来测试 卫星固支情况下整星角位移。

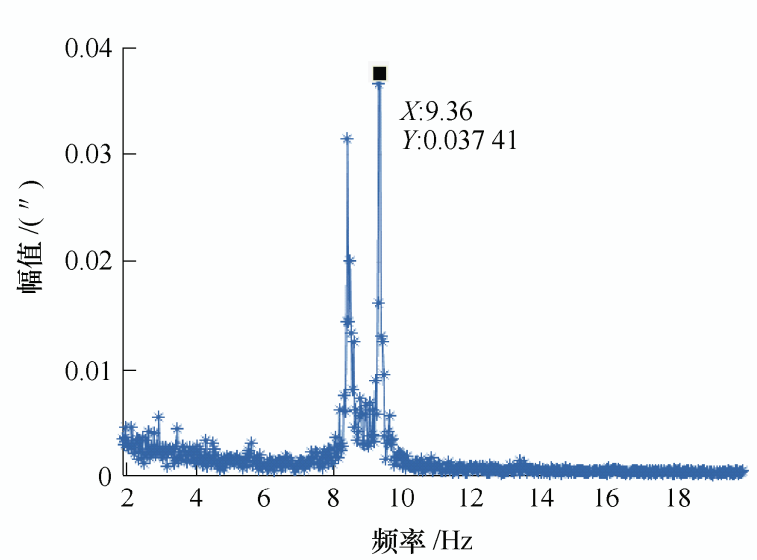

(a) 背景噪声下整星角位移

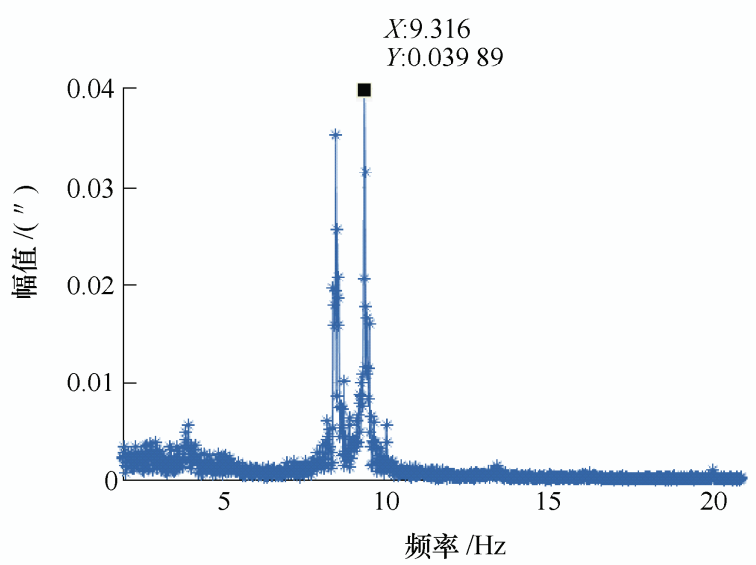

(b) $Y$ 轴飞轮 $600 \mathrm{r} / \mathrm{min}$ 整星角位移 


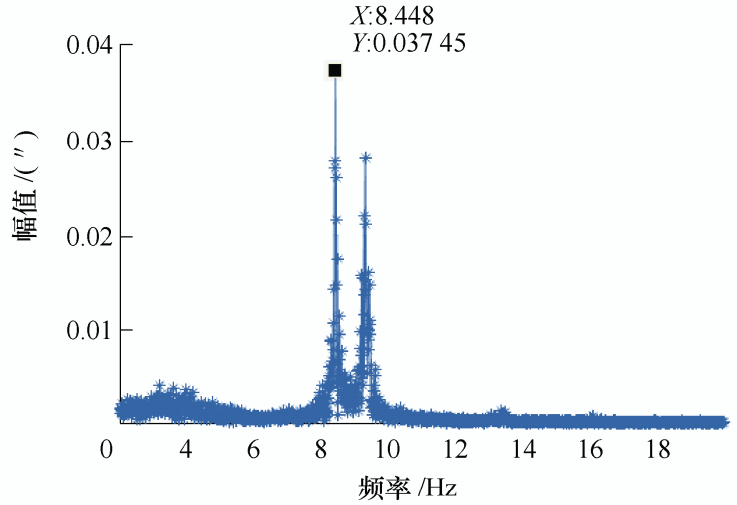

(c) $Z$ 向飞轮转速为 $1200 \mathrm{r} / \mathrm{min}$ 时整星角位移

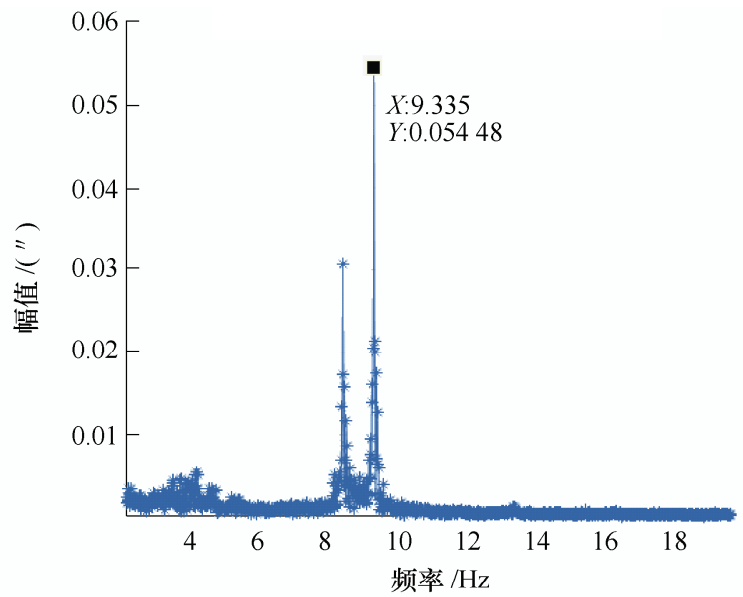

(d) $Z$ 向飞轮转速为 $1550 \mathrm{r} / \mathrm{min}$ 时整星角位移

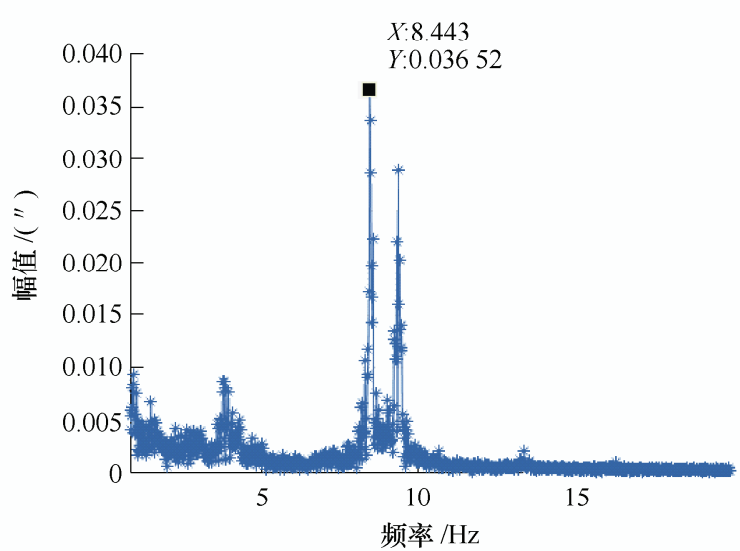

(e) $Z$ 向飞轮转速为 $2078 \mathrm{r} / \mathrm{min}$ 时整星角位移

图 13 卫星固定状态下整星角位移

根据以上工况测得卫星在固支状态下的角位 移。从测量结果的频谱分析可知, 如图 13 所示, 固 支状态下: (1) 在各种背景噪声的影响下整星的角位 移为 0.03741 "(图 13a); (2) $Y$ 向飞轮以 $600 \mathrm{r} / \mathrm{min}$ 单

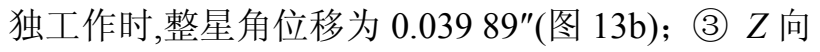
飞轮以 $1200 \mathrm{r} / \mathrm{min}$ 的转速单独工作时, 整星角位移 为 $0.03745^{\prime \prime}$ (图 13c); (4) $Z$ 向飞轮以 $1550 \mathrm{r} / \mathrm{min}$ 的 转速单独工作时, 整星角位移为 0.05448 "(图 13d); (5) $Z$ 向飞轮以 $2078 \mathrm{r} / \mathrm{min}$ 的转速单独工作时, 整星 角位移为 0.03652 "(图 13e)。我们可以看出, 与卫 星悬吊状态下的结果相比, 飞轮在工作过程中在 5 $\mathrm{Hz}$ 处无峰值, 可以证明卫星在悬吊状态下低频处 响应是由于悬吊引起的。频谱分析发现在固定状态 下, 背景噪声为 $9 \mathrm{~Hz}$ 左右, 飞轮在工作过程中同 样在 $8 \sim 9 \mathrm{~Hz}$ 处有颤振响应, 与背景噪声比较契 合, 说明卫星在固定状态下的颤振响应是由背景噪 声引起的。

综上所述, 试验测得整星悬吊状态, $Z$ 轴飞轮 转速为 $1200 \mathrm{r} / \mathrm{min} 、 Y$ 轴飞轮转速为 $600 \mathrm{r} / \mathrm{min}$ 工况 下整星角位移为 0.08932 ", 仿真计算得出物空间角 位移为 $4.806 \times 10^{-7} \mathrm{rad}$ 即 0.09926 "(表 1), 计算可
得其相对误差 $\alpha$ 为 $11.13 \%$, 整星 LOS 角位移仿真 值与试验值的绝对值为 0.009 94", 小于 $0.01 "$, 初步认为在轨遥感图像质量不会受微振动影响 而退化。

\section{表 4 整星 LOS 角位移试验与仿真结果比较}

\begin{tabular}{cccc}
\hline 仿真结果/(") & 试验结果/(") & 相对误差 $\alpha(\%)$ & 绝对值/(") \\
\hline 0.09926 & 0.08932 & 11.13 & 0.00994 \\
\hline
\end{tabular}

\section{5 结论}

本文针对某高分辨率光学卫星在飞轮微振动作 用下产生视轴颤振误差的问题，建立了整星的结构 动力学模型并仿真分析了在飞轮实际工作过程中光 学卫星的视轴误差, 对整星进行了地面飞轮微振动 测试试验。

由 LOS 仿真计算结果可知整星光学系统中主 镜与次镜对视轴误差的贡献最大, 主镜的刚体位移 是造成光学系统产生视轴误差的主要原因, 整星第 8 和 9 阶模态对 LOS 的贡献值最大, 即主镜与次镜 在 $Y$ 方向与 $Z$ 方向的刚体位移对视轴颤振的影响最 
大, 可优化主镜与次镜相关参数或者设置隔振系统 降低主次镜对光学成像系统的影响, 保证成像质量。 卫星在悬吊状态下会产生一个低频振动, 大约为 5 $\mathrm{Hz}$ 左右。随后测量卫星在固定状态下飞轮扰动导致 的颤振情况, $5 \mathrm{~Hz}$ 处无振动, 只在 $9 \mathrm{~Hz}$ 附近产生 振动, 与背景噪声下产生的振动相符, 可以确定悬 吊产生的低频信号时由于卫星刚性悬吊引起的。整 星 LOS 角位移的仿真计算结果与试验结果相比, 其 相对误差 $\alpha$ 为 $11.13 \%$, 绝对值为 0.009 94", 小于 $0.01 "$, 初步认为光学遥感卫星在轨工作时图像质量 不会受微振动影响而退化, 对于光学遥感卫星的微 振动设计具有参考性意义。

\section{参 考 文 献}

[1] KEITH B , VICTOR L , GREGORY J. Integrated opto-mechanical analysis [M]. Washington: National Defense Industry Press, 2015.

[2] ZHANG Zhe, AGLIETTI G, ZHOU Weiyong. Microvibrations induced by a cantilevered wheel assembly with a soft-suspension system[J]. AIAA Journal, 2011, 49(5): 1067-79.

[3] NAGABHUSHAN V, FITZ-COY N. On-orbit jitter control in momentum actuators using a three-flywheel system [J]. Acta Astronatica, 2014, 95: 61-81.

[4] 黄庭轩, 张尧, 徐世杰. 飞轮隔振平台组合系统的动力学 建模[J]. 北京航空航天大学学报, 2013, 39(1): 120-125. HUANG Tingxuan, ZHANG Yao, XU Shijie. Dynamics modeling of flywheel vibration isolation platform combination system[J]. Journal of Beijing University of Aeronautics and Astronautics, 2013, 39(1): 120-125.

[5] 关新, 崔颖慧, 梁鲁, 等. 飞轮隔振装置研制及试验验 证 $[\mathrm{C}] / /$ 高分辨率遥感卫星结构振动及控制技术研讨 会. 湖南, 长沙. 2011：38-44.
GUAN Xin, CUI Yinghui, LIANG Lu, et al. Analysis method on effect of jitter on high resolution agile satellite imaging [C]// Seminar on Structural Vibration and Control Technology of High Resolution Remote Sensing Satellites. Hunan, Changsha. 2011: 38-44.

[6] 李林, 王栋, 徐婧, 等. 飞轮组件微振动对高分辨率光学 卫星光轴的影响[J]. 光学精密工程, 2016, 34(10): 25152522.

LI Lin, WANG Dong, XU Jing, et al. Influence of micro-vibration of flywheel components on optical axis of high-resolution optical satellites[J]. Optics and Precision Engineering, 2016, 34(10): 2515-2522.

[7] KIM D. Micro-vibration model and parameter estimation method of a reaction wheel assembly[J]. Journal of Sound and Vibration, 2014，333(18): 4214-4231.

[8] LEE D, YOON J, HAN J, et al. Integrated framework for jitter analysis combining disturbance, structure, vibration isolator and optical model[C]// Proceedings of SPIE, 2012.

[9] 张博文, 王小勇, 胡永力. 微振动对高分辨率空间相机 成像影响的集成分析 [J]. 航天返回与遥感, 2012,33(2): 60-66.

ZHANG Bowen, WANG Xiaoyong, HU Yongli. Integrated analysis on effects of micro-vibration high resolution space camera imaging[J]. Space Revolving and Remote Sensing, 2012, 33(2): 60-66.

[10] GENBERG V, MICHELS G, DOYLE K, et al. Integrated modeling of jitter MTF due to random loads[C]// Proceedings of SPIE, 2011.

作者简介: 刘瑞婧, 女, 1994 年出生, 博士研究生。主要研究方向为微 振动的光机集成分析。

E-mail: 1418444530@qq.com

李宗轩(通信作者), 男, 1986 年出生, 博士, 副研究员。主要研究方向 为空间光学仪器设计与集成分析。

E-mail: lizongx@126.com 